\title{
Fin des quotas laitiers, contractualisation et stratégies productives : enseignements d'une modélisation bioéconomique
}

\author{
B. LELYON', V. CHATELLIER ${ }^{2}, K$. DANIEL ${ }^{2,3}$ \\ ${ }^{1}$ Institut de l'Elevage, Département Economie, F-75012 Paris, France \\ 2 INRA, UR1134 (LERECO), F-44316 Nantes, France \\ 3 ESA, PRES UNAM Université, ESA LARESS, F-49007 Angers, France \\ Courriel : baptiste.lelyon@idele.fr
}

La suppression du régime des quotas laitiers à horizon 2015 constitue un tournant majeur pour la filière laitière européenne et française. Ce passage d'une régulation publique par des quotas, à une régulation privée par des contrats entre les industriels et les producteurs, est susceptible d'engendrer une augmentation des volumes de production, une baisse du prix du lait et une concentration territoriale de l'offre. A l'échelle des exploitations laitières de l'Ouest de la France, une telle évolution pourrait également se traduire par une spécialisation accrue et une intensification des systèmes de production.

Dans les propositions législatives de réforme de la Politique Agricole Commune (PAC) du 11 octobre 2011, la Commission Européenne a entériné le fait que les quotas laitiers seraient supprimés à l'horizon 2015 (Commission européenne 2011), soit près de trente années après leur mise en œuvre. Cette décision a été prise en considérant que ceux-ci ne sont plus adaptés au contexte actuel de l'économie laitière, caractérisé par une augmentation rapide de la demande internationale et par un développement soutenu des échanges (Colman 2000). En France, probablement plus que dans d'autres pays, la suppression des quotas laitiers suscite à la fois des craintes (baisse du prix du lait, concurrence entre régions, pression sur l'environnement...) et des espoirs (développement de la production, renforcement de la compétitivité...). Au sein de la filière laitière française, il existe un consensus autour de l'idée que les quotas laitiers ont joué, du moins jusqu'à une période récente, un rôle de stabilisation du prix du lait payé aux producteurs. De même, nombreux sont ceux qui considèrent qu'ils ont eu un impact positif sur l'aménagement du territoire et l'environnement (Kroll et al 2010). Outre ce changement de cap dans la régulation de l'offre, le débat engagé sur l'avenir de la filière s'inscrit dans un contexte devenu plus difficile du fait de l'accentuation récente de la volatilité des prix des produits agricoles et des intrants.
L'objectif de cet article est d'analyser en quoi la suppression des quotas laitiers est susceptible d'influencer les stratégies productives des exploitations laitières. En prenant une hypothèse de régulation de l'offre parmi d'autres (il n'existe pas encore de vision partagée sur cette question entre les acteurs de la filière), il a été considéré ici que la suppression des quotas laitiers laisserait place à l'instauration de contrats de type «double volume et double prix» entre les industriels et les producteurs. De manière plus précise, il a été considéré que ces contrats seraient articulés en deux volets : le premier assurerait aux producteurs un débouché stable pour un volume de lait équivalent à leur quota historique (volume $\mathrm{A}$ ) et à un niveau de prix conforme à une tendance de long terme ; le second donnerait la possibilité aux seuls producteurs volontaires de produire plus de lait (volume $\mathrm{B}$ ), mais à un prix inférieur et de surcroît plus variable.

Pour évaluer les effets supposés d'un tel système de régulation de l'offre, un modèle de type bioéconomique a été construit en s'appuyant sur quatre types d'exploitations laitières fréquemment rencontrés dans l'Ouest de la France. Basée sur la programmation mathématique, ce modèle permet de simuler les choix de production des éleveurs en supposant qu'ils cherchent à maximiser leur revenu tout en tenant compte d'un ensemble de contraintes, qu'elles soient biologiques, techniques, structurelles, économiques et réglementaires. Il permet également d'intégrer à l'optimisation du revenu le fait que les agriculteurs ont de l'aversion pour le risque (lié à la volatilité des prix). Ce modèle permet de discuter, à l'échelle de l'exploitation, des implications potentielles du scénario étudié, en termes de combinaisons productives (évolution des équilibres entre les productions), de niveau d'intensification (surfaces de maïs fourrage $v s$ de prairies, chargement par hectare, intensité du recours aux aliments concentrés...) ou de pression environnementale (rejets azotés).

\section{1 / Le modèle bioécono- mique}

La programmation mathématique est une technique permettant de représenter le fonctionnement d'une exploitation agricole soumise à un ensemble de contraintes. Cette technique est utile pour étudier les effets de seuil et pour calculer les rendements marginaux des facteurs de production. Elle permet, en outre, de prendre simultanément en compte des informations relatives à la production, aux prix et aux instruments de la PAC. Le modèle utilisé est constitué de trois éléments de base : $i$ ) une fonction objectif qui maximise le 
revenu ; ii) une description des activités présentes au sein du système, avec les coefficients représentant leurs réponses productives ; iii) un ensemble de contraintes définissant les conditions d'exploitation et les limites du modèle et de ses activités.

\section{1 / Une optimisation du revenu prenant en compte le risque de variation des prix}

La construction de ce modèle bioéconomique répond à l'ambition de représenter, aussi finement que possible, le mode de fonctionnement d'une exploitation laitière. Cela impliquait de traiter aussi précisément que possible la question déterminante des interactions entre les productions animales et les productions végétales. Cela est d'autant plus nécessaire que les exploitations laitières de l'Ouest de la France disposent généralement d'une forte autonomie fourragère et sont souvent diversifiées : $35 \%$ des exploitations laitières spécialisées de l'Ouest de la France (dont le chiffre d'affaire provient pour plus de $50 \%$ de la production laitière) disposent d'un atelier complémentaire à la production de lait (Chatellier et al 2008). Pour représenter cette diversification, plusieurs activités complémentaires à la production laitière, telles que les grandes cultures (blé, maïs grain, colza et pois) et l'engraissement de jeunes bovins, ont été considérées. De même, le modèle propose, pour une production agricole donnée, plusieurs itinéraires techniques alternatifs. Il a donc été construit de telle sorte que l'agriculteur puisse choisir le mode de production le plus pertinent en fonction des contraintes de son exploitation.

La fonction «objectif» placée au cœur de ce modèle vise à optimiser le plan de production (c'est-à-dire la combinaison optimale des différentes productions et des quantités d'intrants utilisées) pour maximiser non pas le revenu, mais l'espérance de l'utilité du revenu (Lelyon et al 2011b). En effet, nombreux sont les agriculteurs qui, en raison de leur aversion au risque, préférèrent adopter une stratégie productive qui favorise l'obtention d'un revenu plus stable, quitte à ce que cela engendre parfois la perte de revenus potentiellement plus élevés. Prendre en compte le risque semblait d'autant plus nécessaire que la volatilité des prix a été particulièrement forte au cours des dernières années. En France, le prix du lait payé au producteur est ainsi passé de $270 € / t$ en 2007 à $380 € / t$ en 2008 , avant de chuter à $220 € / \mathrm{t}$ au printemps de 2009 pour ensuite remonter à 350 $€ / t$ en été 2011 . Le prix des céréales a suivi des évolutions aux intensités comparables.

Tableau 1. Prix des produits et des intrants utilisés dans le modèle (et leur variation autour de cette moyenne).

\begin{tabular}{|l|c|c|}
\hline & Prix & Variation (\%) \\
\hline Lait $(€ / \mathrm{L})$ & 0,29 & \pm 10 \\
\hline Viande (vaches de réforme) & 2,60 & \pm 20 \\
\hline Viande (Jeunes Bovins) & 2,90 & \pm 20 \\
\hline Cultures de ventes & & \pm 30 \\
\hline Blé $(€ / \mathrm{kg})$ & 0,18 & \pm 30 \\
\hline Maïs $(€ / \mathrm{kg})$ & 0,18 & \pm 30 \\
\hline Colza $(€ / \mathrm{kg})$ & 0,30 & \pm 30 \\
\hline Pois $(€ / \mathrm{kg})$ & 0,17 & \pm 30 \\
\hline Concentrés de production $(€ / \mathrm{kg})$ & 0,34 & \pm 30 \\
\hline Tourteau de soja $(€ / \mathrm{kg})$ & 0,32 & \pm 30 \\
\hline Tourteau de colza $(€ / \mathrm{kg})$ & 0,20 & \pm 30 \\
\hline Ammonitrate $(€ / \mathrm{kg})$ & 0,25 & \\
\hline
\end{tabular}

Pour intégrer cet aspect, le prix des produits agricoles et des intrants est variable et suit une loi normale dont la moyenne et l'écart-type ont été calculés en fonction de l'évolution constatée des prix entre 2006 à 2010 (tableau 1). Cela se traduit par différents niveaux de revenus probables pour une même combinaison d'activités. Pour pénaliser les activités procurant des revenus risqués, nous mettons ces revenus sous une forme exponentielle négative (Flaten et Lien 2007). Ainsi, le modèle ne maximise pas le revenu de l'activité, mais l'utilité générée par ce revenu : chaque euro de revenu supplémentaire procurera une utilité de plus en plus faible (rendement marginal décroissant). L'agriculteur cherche ainsi à maximiser son revenu tout en minimisant sa variabilité.

D’une façon détaillée, le revenu (il s'agit ici plus précisément de l'Excédent Brut d'Exploitation (EBE) est déterminé de la manière suivante :

$\mathbf{E B E}=$ Produit lait + Produit viande + Produit cultures + Droit à Paiement Unique (DPU ou aides découplées de la PAC)

- Intrants liés aux productions végétales et aux productions animales (semences, fertilisation, traitements, travaux par tiers, concentrés, contrôle laitier, frais vétérinaires, frais d'élevage et minéraux)

- Coûts fixes (carburants, entretiens matériel et bâtiments, eau, électricité)

Dans ce modèle, l'ensemble des variables physiques (exprimées en quantité) sont représentées et font l'objet de l'optimisation de façon à déterminer la combinaison productive conduisant au niveau le plus élevé d'EBE. Le modèle intègre la dynamique de renouvellement du troupeau en dissociant différents types d'animaux selon leur âge (veau, génisse, vache, taurillon). Concernant les cultures, le modèle offre l'opportunité de cultiver soit de la prai- rie et du maïs pour les fourrages et du blé, du maïs grain, des pois et du colza pour les cultures de vente. Les animaux peuvent ainsi consommer de l'ensilage de maïs, de l'ensilage d'herbe, du foin et de l'herbe pâturée. Les concentrés proposés aux animaux sont le tourteau de colza, le tourteau de soja, un concentré de production (1,3 UFL et $150 \mathrm{~g}$ de $\mathrm{PDI} / \mathrm{kg})$. Il est également possible de recourir à l'autoconsommation du blé produit sur l'exploitation et d'utiliser le lait produit sur l'exploitation pour l'alimentation des veaux. Les achats de fourrages ne sont, en revanche, pas permis, considérant que les éleveurs laitiers de l'Ouest de la France sont autonomes sur ce plan.

\section{2 / Les principales caractéris- tiques du modèle}

Dans l'objectif de représenter un fonctionnement aussi réaliste que possible d'une exploitation laitière, ce modèle comporte quatre caractéristiques principales :

- Une saisonnalité de la production de l'herbe et la prise en compte des pics de travail. L'année civile est décomposée en quatre périodes équivalentes (proche des quatre saisons : printemps, été, automne et hiver). La prise en compte de la saisonnalité est utile pour intégrer les variations saisonnières relatives à l'utilisation des prairies. En effet, le potentiel de croissance de l'herbe et sa valeur alimentaire varie de manière importante d'une saison à l'autre (Berentsen et al 2000). La périodisation du modèle permet, en outre, d'intégrer la contrainte de travail en attribuant à chaque activité des besoins en main-d'œuvre qu'il est possible de moduler selon les saisons (Bewley et al 2001). Cela concerne aussi bien les cultures (travaux de semis et de récolte) que les vaches laitières (vêlage et mise à l'herbe).

- Une flexibilité de la production laitière. Les éleveurs ont la possibilité, via les options prises pour le rationnement 
Tableau 2. Données techniques et structurelles des quatre cas-types d'exploitations.

\begin{tabular}{|l|c|c|c|c|}
\hline & $\begin{array}{c}\text { Lait } \\
\text { Extensif }\end{array}$ & $\begin{array}{c}\text { Lait } \\
\text { Sem i-intensif }\end{array}$ & $\begin{array}{c}\text { Lait + Céréales } \\
\text { Intensif }\end{array}$ & $\begin{array}{c}\text { Lait + Jeunes } \\
\text { Bovins Intensifs }\end{array}$ \\
\hline Surface Agricole Utile (ha) & 78 & 50 & 137 & 100 \\
\hline Surface en prairie permanente (ha) & 42 & 15,5 & 14 & 10 \\
\hline Quota (L) & 285000 & 290000 & 460000 & 400000 \\
\hline Unité de Travail Agricole (nb.) & 1,7 & 1,5 & 2,0 & 2,7 \\
\hline Capacité des bâtiments (nb. de têtes) & 62 & 37 & 59 & 122 \\
\hline Taux de fécondité (\%) & 96 & 90 & 90 & 90 \\
\hline Taux de renouvellement (\%) & 25 & 35 & 37 & 40 \\
\hline Potentiel laitier des vaches (Lan) & 6000 & 8500 & 8500 & 9000 \\
\hline Rendement des cultures (t MS/ha/an) & & & & \\
\hline Blé & 6,1 & 8,1 & 8,1 & 8,1 \\
\hline Maïs grain & - & - & 10,0 & - \\
\hline Colza & - & - & 3,8 & - \\
\hline Pois & - & - & 5,0 & - \\
\hline Maïs ensilage & 10,2 & 12,2 & 15,2 & 14,2 \\
\hline Ensilage d'herbe & 8,5 & 8,5 & 8,5 & 8,5 \\
\hline Pâturage & 8,5 & 7,0 & 6,0 & 6,0 \\
\hline Foin & 8,5 & 7,5 & 7,5 & 7,5 \\
\hline Poids des carcasses (réforme) $(\mathrm{kg})$ & 375 & 325 & 325 & 325 \\
\hline
\end{tabular}

des bovins, de faire varier le rendement laitier. Le rendement laitier suit les lois de réponses classiques, à savoir 0,44 UFL et 48 g de PDI par litre de lait produit, jusqu'à atteindre le niveau de production moyen constaté sur chaque système type (voir tableau 2). Le niveau de production moyen représente la quantité de lait que peut produire un animal nourri avec une ration équilibrée, mais sans être suralimenté. Toutefois, dans la pratique, le rendement laitier peut dépasser ce niveau de production moyen dès lors que la ration est suffisamment riche en nutriments. Les éleveurs ont parfois recours à un usage plus important de concentrés pour produire un volume supplémentaire (Faverdin et al 2007), comme cela a été le cas début 2008 et au cours de l'été 2011. Brun-Lafleur et al (2010) montrent que le rendement laitier, s'il est linéaire jusqu'à un certain point, suit un rendement décroissant dès lors qu'il dépasse le niveau de production moyen de l'animal. Les besoins alimentaires pour la production d'un litre de lait supplémentaire au dessus du niveau de production moyen sont ainsi trois fois plus importants.

- Un système alimentaire basé sur les besoins physiologiques des animaux. Une attention particulière a été portée aux rations des bovins. La quantité d'aliment ingérée par animal et par jour est déterminée en fonction : $i$ ) des besoins nutritionnels en énergie et en protéines; et ii) de la valeur alimentaire des fourrages et des concentrés exprimés selon le système UFL-PDI (INRA 2007). Les besoins totaux du troupeau en fourrages ne doivent pas être supé- rieurs aux rendements fourragers des cultures et chaque animal ne peut ingérer une quantité de fourrages supérieure à sa capacité d'ingestion.

- La multi-production des prairies et la fertilisation des cultures. Ce modèle intègre la particularité de la prairie qui, à partir de la biomasse produite, peut fournir différents types de fourrage : pâturage, foin, ensilage d'herbe. La répartition entre ces différentes productions est optimisée par le modèle. En outre, le rendement des cultures (prairies, fourrages et céréales) dépend de la quantité d'azote utilisée (Godard et al 2008).

Comme dans tous les modèles de ce type, le système de production global est soumis à un ensemble de contraintes qui pèsent sur les choix de l'agriculteur. Les contraintes structurelles définissent les caractéristiques générales de l'exploitation (surface agricole, quota laitier, nombre d'emplois agricoles). Concernant les cultures, le modèle prend en compte les contraintes de rotation et veille à une succession cohérente des cultures (cultures d'hiver et de printemps). Les contraintes environnementales sont de trois types : i) le respect de la directive «nitrates», à savoir que la quantité d'azote organique par hectare ne peut excéder le seuil de $170 \mathrm{~kg}$; ii) le maintien obligatoire des prairies âgées de plus de 5 ans; iii) une Prime Herbagère Agro-Environnementale (PHAE) de $75 € /$ ha est accordée, si les surfaces en prairie représentent plus de $75 \%$ de la Surface Agricole Utile (SAU) et si le taux de chargement est inférieur à 1,4 UGB herbivore par hec- tare de Surface Fourragère Principale (SFP).

De manière à maximiser l'EBE, le modèle détermine ainsi le nombre de bovins de chaque type, les quantités ingérées en fourrages et en concentrés pour chaque catégorie de bovins, le rendement laitier par vache, la surface des différents types cultures (prairie temporaire, maïs...) la quantité de céréales vendues et/ou autoconsommées, le rendement des cultures (fourrages et céréales) et leur fertilisation.

\section{3 / Un modèle appliqué à qua- tre cas-types d'exploitations lai- tières}

Ce modèle est appliqué à quatre castypes d'élevages laitiers représentatifs d'une partie de la diversité des systèmes rencontrés dans l'Ouest de la France (tableau 2). Si la structure du modèle est unique, les coefficients techniques varient en fonction de ceux-ci. Les données utilisées sont issues de l'enquête annuelle de l'Institut de l'Elevage (2009) et du Réseau d'Information Comptable Agricole (RICA).

- Spécialisé lait extensif. Il correspond à un système d'élevage extensif, éligible à la PHAE, où les prairies prédominent très largement, tant dans l'assolement que dans le système alimentaire.

- Spécialisé lait semi-intensif. Il adopte une stratégie plus productive, en raison notamment d'une moindre abondance de foncier. Les vaches laitières, dont le rendement laitier est supérieur au cas-type précédent, sont alimentées pour une part avec du maïs fourrage. 
Cette plus grande intensification permet de libérer un peu de surface pour la production de céréales.

- Le système laitier intensif avec une diversification vers la production de céréales. Il bénéficie d'une structure plus conséquente avec 137 hectares et une production laitière de 460000 litres par an. L'intensification des productions fourragères est privilégiée pour favoriser le développement de la production céréalière.

- Le système laitier intensif avec une diversification vers la production de jeunes bovins (jusqu'à 80 têtes par an). Dans le même esprit que le cas-type précédent, l'intensification de l'atelier laitier permet de libérer des productions fourragères pour l'alimentation des jeunes bovins (production qui offre 1'opportunité de mieux valoriser économiquement les veaux mâles nés sur l'exploitation).

\section{2 / Un scénario «double volume et double prix» pour l'après 2015}

Après une présentation des options possibles pour réguler l'offre dans une situation où les quotas laitiers auront disparu, des précisions sont apportées sur le scénario testé ici à partir du modèle dont les caractéristiques ont été présentées précédemment.

En l'absence de quotas laitiers, l'instauration d'une contractualisation avec les producteurs de lait est nécessaire pour permettre aux industriels de sécuriser leurs approvisionnements, en quantité et en qualité. Les entreprises n’ont généralement pas intérêt à collecter plus de lait que ce que leurs usines sont capables de transformer et, plus encore, que ce que leurs débouchés autorisent. Lorsque la collecte devient supérieure aux capacités de transformation et/ou de commercialisation, le lait collecté est parfois revendu immédiatement sur le marché «spot» à un prix inférieur au prix d'achat. Il en résulte des pertes économiques importantes. Dans un tel cadre, certaines entreprises entendent instaurer un système de prix différenciés suivant la destination finale des produits laitiers ; en France, cette stratégie est soutenue par les structures coopératives et un groupe privé (Institut de l'Elevage 2011). Dans ce schéma, un volume "A» serait attribué aux producteurs sur la base de tout ou partie du quota laitier historique et moyennant le prix d'équilibre constaté sur le marché intérieur (prix «A») ; un volume «B», optionnel, pourrait être accordé aux producteurs, mais pour un niveau de prix plus faible et indicé sur l'évolution du prix des produits industriels (beurre et poudres). Un tel dispositif permettrait aux industriels de devenir plus réactifs et plus compétitifs sur des marchés concurrentiels (produits à faible valeur ajoutée ou les marchés d'exports). Cependant, le volume contractuel de base (Volume A) n'est pas envisagé de la même façon par tous les transformateurs, certains considèrent qu'il sera égal au quota historique (dernière référence connue en 2015) alors que d'autres souhaitent l'ajuster à la baisse afin que ce Volume A corresponde au plus près au débouché de produits à forte valeur ajoutée.

Cette option qui consisterait à adopter des prix différenciés est toutefois critiquée par certains acteurs de la transformation laitière (en France, essentiellement ceux issus du secteur privé). Ils considèrent qu'un tel système est difficilement applicable en raison de la difficulté technique de procéder à une segmentation efficace entre les volumes A et $\mathrm{B}$; or le problème de l'étanchéité peut inciter à des fraudes. De plus, ils anticipent que les centrales d'achat pourraient faire pression à la baisse sur les prix issus du volume A. Ils soulignent surtout le fait qu'un tel système entraînerait une perte de compétitivité à long terme de la filière laitière. Au travers de la baisse des prix et de l'augmentation des volumes, les entreprises de la transformation ont, en effet, la possibilité de reporter aux producteurs (qui deviennent alors responsables) leurs propres défaillances en termes d'innovations et de structure de coûts, etc.

Comme y invite la Commission européenne, l'instauration de contrats entre les producteurs de lait et les industriels est nécessaire pour clarifier les relations existantes et permettre aux différentes parties de bénéficier d'une visibilité à moyen et long terme. Ces futurs contrats ne mentionneront cependant pas le niveau de prix que les producteurs de lait obtiendront au cours de la période considérée. Le prix moyen dépendra d'abord de l'équilibre futur atteint entre l'offre et la demande. Les principaux enjeux de la négociation entre les producteurs et les industriels porteront donc notamment sur : la mise en place ou non de contrats collectifs (interrégionaux); les modalités de rémunération du lait produit en volume A et B ; le transfert de propriété du lait à une organisation de producteurs chargée de négocier collectivement le prix du lait ; la méthode de planification de la production laitière à l'échelle individuelle ou collective (et les pénalités associées à un éventuel non-respect) ; les modalités de transmission d'un contrat d'un agriculteur à un autre, etc.
En l'absence d'une politique de contingentement de l'offre, le risque d'une baisse du prix du lait existe, ce d'autant que l'élasticité de la demande par rapport au prix est, dans ce secteur, plutôt faible. Plusieurs modèles d'équilibres partiels ont été construits pour évaluer l'impact de la suppression des quotas sur le niveau des prix au sein de l'Union Européenne (INRA et University of Wageningen Consortium 2002, Bouamra-Mechemache et al 2009). Ils ont démontré qu'une augmentation de la production laitière européenne comprise entre 7 et $10 \%$ était susceptible de provoquer une baisse des prix payés aux producteurs de l'ordre de 21 à $26 \%$.

La dynamique future du prix du lait dépendra cependant de nombreux facteurs dont celui de l'éventuelle contractualisation sur les volumes entre les entreprises de la transformation du lait et les producteurs. Les entreprises (privées ou coopératives) seront incitées à prendre le relais de la puissance publique dans la gestion de l'offre. Si ces politiques contractuelles, menées au sein de chaque entreprise, s'acheminent vers une gestion rigoureuse de l'offre collective, la baisse du prix du lait payé au producteur pourrait être moins intense que celle pressentie dans les modélisations théoriques.

Dans le scénario de sortie des quotas testé ci-après, il est considéré qu'un système de contractualisation du type «double volume et double prix» est mis en place par les entreprises, dans le cadre d'un accord passé avec les producteurs. Deux volumes de production distincts sont ainsi considérés : i) chaque producteur de lait bénéficie d'un volume de production «A» fixe (équivalent à son quota historique) et dont le prix à la tonne est également fixe et prédéterminé (il est fixé, pour l'exemple ici, à $290 € / \mathrm{t}$ ) ; ii) de manière complémentaire, chaque producteur de lait a la possibilité de produire un volume de lait supplémentaire (dit «volume B»), limité cependant ici dans les simulations à l'équivalent de $30 \%$ du volume $\mathrm{A}$. Ce volume «B» est payé à un prix non seulement plus faible que le volume «A», mais également variable. Ainsi, d'après les travaux de BouamraMechemache et al (2009) une augmentation de la production laitière de $1 \%$ entraîne une diminution du prix du lait en volume $\mathrm{B}$ de $3 \%(\alpha=3)$. Le prix est donc déterminé de la façon suivante :

$$
\begin{gathered}
\text { Prix lait Volume } B= \\
\text { Prix Volume A } \times\left(1-\left(\frac{\text { Volume } B}{\text { Quota historique }}\right) \times a\right)
\end{gathered}
$$

Le modèle étant construit à l'échelle de l'exploitation, nous considérons dans 
le scénario testé que chaque exploitation intervient seule sur le marché. De ce fait, chaque exploitation obtiendra un prix du lait payé pour le volume B différent des autres exploitations. L'agrégation des quatre types d'exploitations pour obtenir un prix commun ne serait pas pertinente car le niveau de représentativité des élevages considérés est insuffisant (il conviendrait de raisonner à une échelle beaucoup plus large, c'est-à-dire en appliquant cette simulation à un nombre beaucoup plus conséquent d'exploitations). Le prix du lait payé en volume B obtenu lors de la simulation représente donc le prix en dessous duquel il n'est plus économiquement rentable de produire du lait en volume B.

Cette simulation est conduite à structure d'exploitation constante. Cela signifie qu'elle ne permet pas de procéder à des investissements (bâtiments, foncier) propices à agrandir les capacités productives (ce qui peut naturellement être envisagé à long terme). Il est cependant admis que le cheptel de vaches laitières puisse augmenter au maximum de $10 \%$ dans la mesure où les bâtiments d'exploitation ne sont pas, du moins pour de nombreuses exploitations, totalement saturés. Deux raisons expliquent ce phénomène : l'amélioration constante du rendement laitier entraîne, à quota laitier constant, une baisse du cheptel de vaches laitières ; des investissements importants ont été réalisés au fil de la dernière décennie pour adapter les bâtiments d'exploitation aux normes environnementales (les producteurs avaient alors anticipé une taille souvent supérieure au potentiel productif du moment).

\section{3 / Les résultats issus de la modélisation et discussion}

Cette troisième section présente les principaux résultats issus du scénario testé de sortie des quotas (double volume et double prix) et discute de la sensibilité de ces résultats aux hypothétiques variations de prix des autres produits agricoles présents dans les exploitations.

\section{1 / Un potentiel de développe- ment de la production laitière}

Les résultats de cette simulation indiquent que certaines exploitations laitières de l'Ouest de la France disposent, à structure constante, d'un potentiel de développement de la production laitière assez important : trois des quatre exploitations présentent une augmentation supérieure à $10 \%$ (tableau 3 ). Ce potentiel de développement tient essentielle- ment au fait que les exploitations peuvent accroître le rendement laitier (en jouant sur le système d'alimentation pour le système Extensif) ou réorienter une partie des surfaces céréalières au profit des cultures fourragères pour les trois autres systèmes. Sans que cela ne soit généralisable, nombreuses sont les exploitations de cette région qui ont connu, au cours des deux dernières décennies, un développement plus rapide de leur surface agricole (par achat ou location) que de leur quota laitier (Ben Arfa et al 2010). Cette situation résulte principalement des règles appliquées en France dans la gestion des quotas laitiers et qui font que le transfert de quotas laitiers est intimement lié au foncier. Pour s'en convaincre, il n'est pas inutile de rappeler que la quantité de lait produite par hectare de SAU est, en moyenne, de seulement 4200 litres par hectare dans l'Ouest de la France contre, par exemple, 8800 litres par hectare au Danemark et 11500 litres par hectare aux Pays-Bas. Dans l'hypothèse où la suppression des quotas laitiers permettrait aux producteurs de l'Ouest de développer la production de lait (ceci supposant que les entreprises de la transformation soient compétitives et capables d'accroître leurs exportations), ceux-ci auraient la possibilité de revisiter leurs choix productifs antérieurs. Ils pourraient ainsi choisir de renforcer leur spécialisation en production laitière en augmentant les surfaces fourragères (au détriment des autres cultures) et en abandonnant (ou en réduisant) certaines activités complémentaires devenues, le cas échéant, économiquement moins rentables (céréales, engraissement des jeunes bovins...).

Grâce à une hausse de la production laitière estimée à $10 \%$ en moyenne, avec d'importants écarts selon les castypes, le scénario testé laisse entrevoir une amélioration moyenne de l'EBE de $5 \%$. La croissance de la production nécessite, en effet, des charges variables supplémentaires, essentiellement en raison de l'augmentation du cheptel (hausse du coût alimentaire global et substitution entre les céréales et les fourrages).

Le système Extensif est celui qui bénéficie économiquement le plus des volumes de production supplémentaires : avec une hausse de la production estimée à $14 \%, 1$ 'EBE progresse de $12 \%$ car les effets de substitution sont ici plus faibles qu'ailleurs. L'augmentation de la production résulte surtout de la hausse du rendement laitier, et non d'une croissance de cheptel. Le prix du lait vendu au titre du volume B est de $177 € / t$ pour le système Extensif, contre près de $200 € / \mathrm{t}$ dans les autres systèmes (moins autonomes). L'augmentation de l'EBE est plus modeste pour les trois autres systèmes (tableau 3) puisqu'ils choisissent d'augmenter l'effectif de vaches laitières plutôt que de distribuer des concentrés alimentaires supplémentaires (pour ainsi dépasser le niveau de production moyen). Ces exploitations doivent donc convertir des surfaces de céréales en surfaces de fourrages pour nourrir ces animaux supplémentaires, ceci entraînant une diminution des recettes céréalières et une hausse des charges alimentaires.

Pour les systèmes semi-intensif et Lait+céréales Intensif, le principal facteur limitant le développement de la production est le nombre de places disponibles dans les bâtiments d'élevage. Pour ces exploitations, l'augmentation de la production de lait passe d'abord par une augmentation du cheptel de vaches laitières. L'amélioration du rendement laitier est, quant à elle, plus difficile à obtenir et elle implique un surcoût alimentaire qui devient assez peu compatible avec le prix du lait payé en volume $B$, puisque chaque litre de lait supplémentaire produit au-delà du niveau moyen de production nécessite trois fois plus de nutriments.

Pour le système Lait + Jeunes bovins Intensif, la situation est différente. Le potentiel de développement de la production laitière est théoriquement important car il est envisageable d'abandonner la production de jeunes bovins (dont les primes ont été intégralement découplées suite à la précédente réforme de la PAC) et d'affecter les surfaces fourragères dédiées à cette production au profit des vaches laitières. Compte tenu des niveaux de prix appliqués (prix du lait en volume B vs prix de la viande bovine) dans la simulation, l'agriculteur a cependant intérêt à conserver cette production. Dans ce cas, une forte croissance de la production laitière entraînerait, il est vrai, une baisse importante du prix du lait payé en volume B. L'introduction du coefficient d'élasticité dans le modèle permet, en effet, de limiter une éventuelle surproduction. Dans sa stratégie d'optimisation, le modèle fonctionne comme si l'éleveur avait conscience qu'une augmentation trop importante de sa production de lait aurait un effet négatif en retour sur le prix. Il cherche donc à obtenir un juste équilibre entre volume et prix. L'augmentation de la production laitière passe donc ici par une légère croissance de cheptel de vaches et par la commercialisation du lait initialement orienté vers l'alimentation des veaux mâles, grâce à l'achat de poudre de lait.

Outre les aspects économiques, cette modélisation permet aussi de tenir compte de l'évolution de la contrainte de travail. Pour les systèmes Extensif et 
Tableau 3. Impacts d'une suppression des quotas laitiers et de la mise en place d'un système de contractualisation du prix du lait sur les résultats technico-économiques des quatre cas-types d'exploitations.

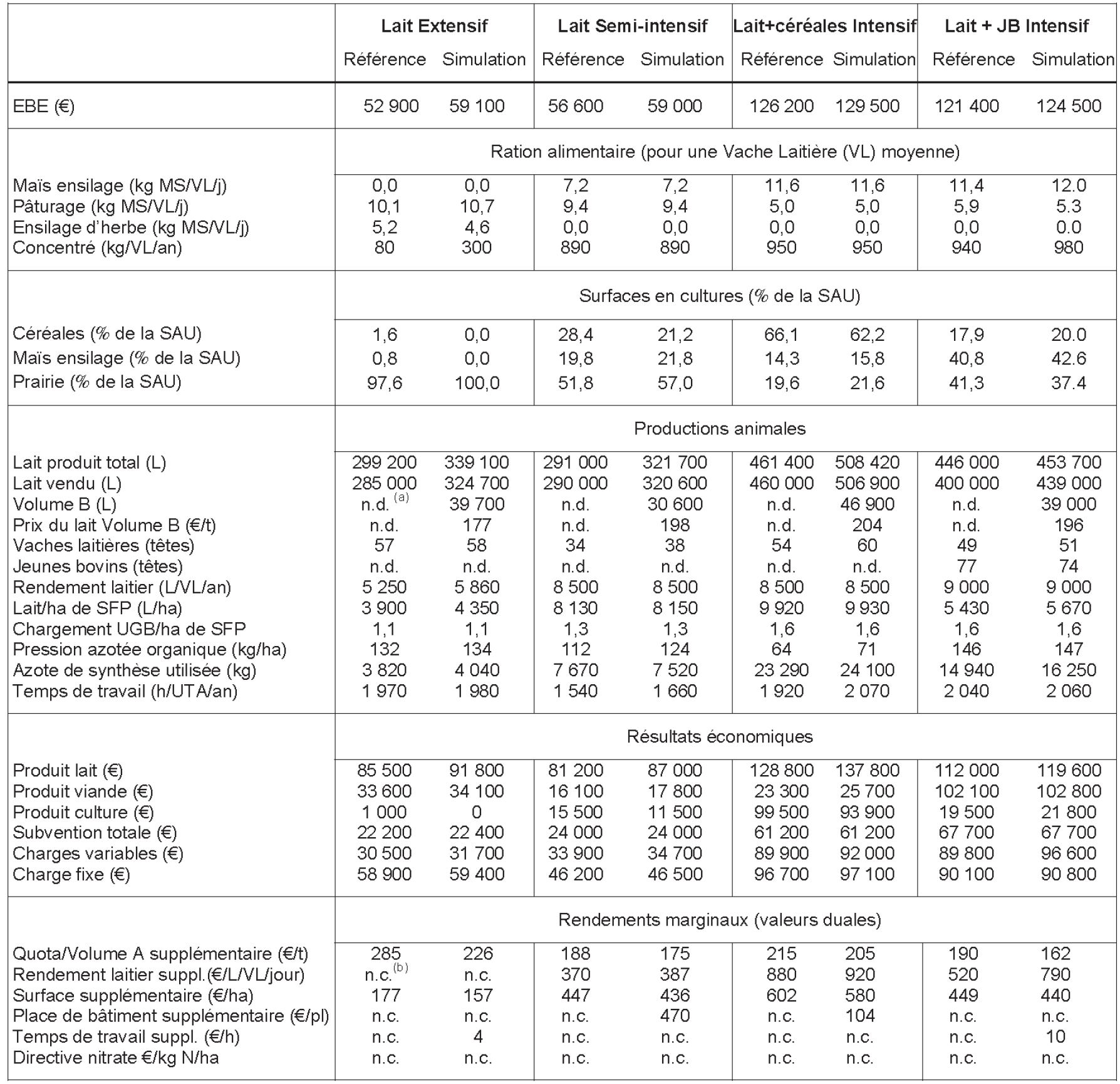

(a) n.d. : non disponible ; (b) n.c. : non contraignant.

Lait+Jeunes Bovins, la quantité de travail supplémentaire n'augmente que marginalement $(+2 \%)$ car l'augmentation de la production ne passe pas ou peu par une croissance du cheptel. Pour les deux autres systèmes, la pression sur la main-d'œuvre est, en revanche, plus conséquente. Ainsi, dans le cas du système semi-intensif, l'amélioration de l'EBE $(+4 \%)$ implique une quantité de travail supplémentaire de $+8 \%$ (respectivement +3 et $+8 \%$ pour le système Lait+Céréales). La flexibilité des systèmes de production est très variable et les moyens à mettre en place pour accroître la production laitière peuvent avoir de forts impacts sur l'organisation de la main-d'œuvre dans les élevages.

\section{2 / Une intensification modérée du système de production}

Selon les résultats de la simulation, l'instauration d'un système «double volume et double prix» pourrait inciter de nombreuses exploitations laitières de l'Ouest à se spécialiser davantage en production laitière et à intensifier leur système de production, dans la limite cependant des seuils imposés par les normes environnementales. Le principal mouvement observé est celui d'un développement de la production fourragère au détriment des surfaces céréalières pour pouvoir augmenter le cheptel laitier. Celui-ci se fait à parts égales entre les surfaces de maïs ensilage et celles de prairies. L'instauration d'un découplage total des aides directes allouées aux superficies de maïs ensilage n'est pas neutre sur l'évolution de la répartition des surfaces fourragères, car les agriculteurs peuvent désormais limiter ou même abandonner la culture du maïs ensilage tout en conservant le 
bénéfice des primes versées historiquement à ce titre. Le découplage des aides qui restent associées à une référence historique offre donc de réelles opportunités pour optimiser les systèmes de production. Ainsi, par exemple, suite à l'instauration du découplage total, le système Extensif a économiquement intérêt à supprimer toutes les surfaces cultivées (Lelyon et al 2011a).

D'après les références utilisées dans les cas-types et les hypothèses formulées dans la simulation, il apparaît que le scénario étudié ne conduit pas la directive nitrate (pression azotée inférieure à $170 \mathrm{~kg} / \mathrm{ha}$ ) à être un facteur limitant de la production puisqu'aucun des systèmes n'atteint ce niveau. Cela ne signifie pas, pour autant, que cette norme environnementale n'est pas amenée à jouer un rôle important dans l'évolution des systèmes productifs de l'Ouest de la France, une fois les quotas laitiers supprimés. Elle contribuera en effet à limiter une intensification excessive de certains systèmes de production, notamment ceux où le foncier est limitant. Si la directive «nitrates» n'est pas bloquante, la simulation met cependant en évidence une accentuation de la pression azotée (jusqu'à $10 \%$ pour les systèmes semi-intensif et Lait+ Céréales) du fait de l'augmentation du cheptel de vaches laitières. De même, elle souligne un recours accentué aux engrais de synthèse (azote) car les agriculteurs entendent améliorer la productivité des surfaces fourragères (particulièrement dans le cas du pâturage). L'intensification du système de production est néanmoins modérée, car le chargement, exprimé au travers du nombre d'UGB herbivores par hectare de SFP et la quantité de lait produite par hectare de SFP demeurent stables.

Sachant que l'augmentation du rendement laitier par vache implique une hausse des apports alimentaires particulièrement importante au-delà d'un certain niveau de productivité, ce critère devient souvent économiquement contraignant pour le développement de la production (voir le rendement marginal dans le tableau 3). Lorsque l'éleveur augmente le niveau de production des vaches d'un litre de lait supplémentaire par jour sur l'ensemble de la lactation, cela génère une hausse globale de l'EBE allant de 390 à $900 €$ selon le cas-type.

Ces simulations montrent, en outre, que le rendement marginal d'un litre de lait supplémentaire en Volume A est positif pour l'ensemble des exploitations (tableau 3). Cette valeur diminue d'environ $10 \%$ avec la mise en place de la contractualisation, mais reste cependant à un niveau élevé. Cela signifie que l'exploitant a toujours un intérêt économique à augmenter le Volume A et qu'il existe donc une rente économique liée à ce volume. Ce phénomène n'aurait pas lieu dans le cadre d'une libéralisation totale du système des quotas laitiers ou les rendements marginaux seraient alors de zéro : les exploitants n'ayant plus de limite administrative à la production. Dans tous les cas, la mise en place d'un système contractuel avec double volume permet aux industriels de bénéficier d'une adaptation de leur approvisionnement à la réalité des marchés concurrentiels, tout en donnant la possibilité aux éleveurs de produire davantage.

\section{3 / Sensibilité de la production de lait en Volume $B$ aux prix des autres activités}

Le prix d'équilibre du lait produit en Volume $B$ déterminé par le modèle reflète, pour chaque système, le niveau à partir duquel l'exploitant est prêt à produire plus, en fonction d'une conjoncture donnée pour les autres productions (céréales et jeunes bovins). C'est pourquoi, les résultats de cette simulation sont désormais discutés en fonction du prix relatif de ces dernières. Le prix des céréales est un élément important des choix productifs, ce d'autant plus qu'il influe sur le prix des concentrés alimentaires (dans cette simulation, chaque variation de prix des céréales de $10 € /$ tonne se traduit également par une variation du prix de l'en- semble des concentrés de $10 € /$ tonne). Une augmentation de celui-ci joue négativement sur la production de lait en Volume B. C'est le cas pour le système Extensif, où une baisse de la production de lait est envisagée afin de contenir l'augmentation des coûts alimentaires (figure 1). La pente de la droite est faible car les possibilités de substitution entre le lait et les céréales sont limitées en raison de faibles rendements céréaliers. La pente de la courbe est, en revanche, plus forte pour les systèmes semi-intensif et Lait+Céréales qui bénéficient de rendements plus élevés et qui ont donc intérêt à substituer le lait valorisé en Volume B par des céréales (à partir de $150 € / \mathrm{t}$ de céréales pour l'élevage semi-intensif). La diminution du volume de lait produit est plus faible pour l'exploitation Lait+Jeunes bovins car le phénomène de substitution s'opère essentiellement entre l'atelier d'engraissement de jeunes bovins et les surfaces de céréales. A partir d'un prix de $150 €$ par tonne de céréales, le modèle considère qu'il n'est plus économiquement souhaitable d'acheter des veaux mâles à l'extérieur; à partir d'un prix de $180 €$ par tonne de céréales, l'activité d'engraissement est considérée comme moins rentable (en raison également de l'augmentation des coûts de concentrés).

Ces résultats confortent les observations réalisées lors du Recensement Agricole de 2010 (Roux 2011) qui révèlent une diminution de près de la

Figure 1. Influence du prix des céréales sur le volume de production laitière supplémentaire (\% du volume A) simulé pour les quatre cas-types d'exploitations.
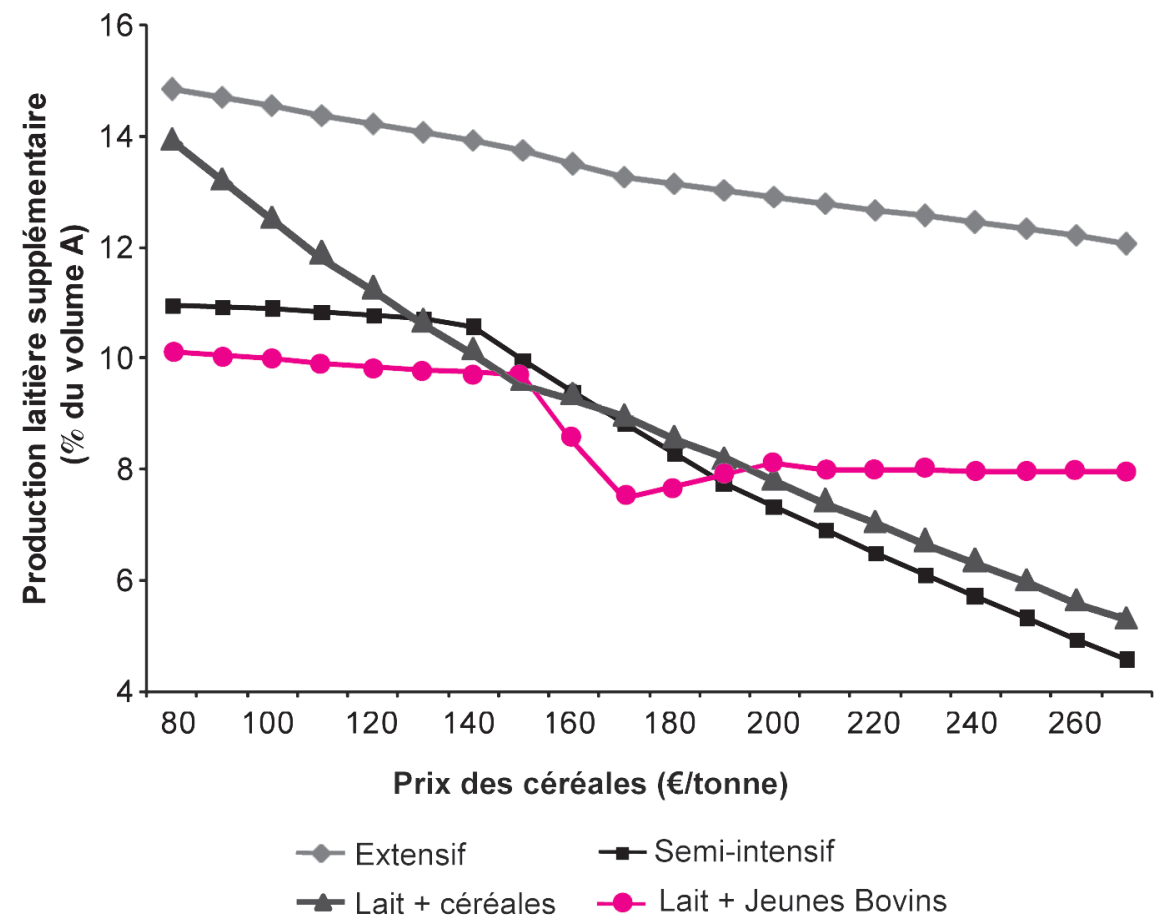
moitié des exploitations spécialisées en lait en dix ans alors que l'effectif d'exploitations céréalières s'est, quant à lui, pratiquement maintenu. Cela traduit un abandon, dans certaines exploitations agricoles, de leur activité bovine au profit des cultures de vente, notamment dans les zones de polyculture élevage (Poitou-Charentes, Picardie...). Toutefois, pour les quatre systèmes de l'Ouest représentés, il ressort que le maintien de la production laitière en Volume A est toujours une priorité des éleveurs, quels que soient les prix considérés (le rendement marginal d'un litre de lait supplémentaire en Volume A reste positif). En effet, les coûts fixes engagés au service de l'activité laitière (dotations aux amortissements et frais financiers) sont souvent trop élevés pour que les éleveurs puissent privilégier la voie d'un abandon total du lait au profit des céréales. Ceci est d'autant plus vrai que la surface agricole des exploitations laitières de l'Ouest est souvent nettement inférieure au seuil de rentabilité rencontré classiquement dans les exploitations spécialisées de grandes cultures.

Outre le prix des céréales, la production de lait réalisée en volume B dépend aussi du prix du lait obtenu en volume A. Ce dernier est non seulement déterminant du chiffre d'affaires total de l'entreprise, mais également de sa rentabilité. Comme cela est vérifié dans le cas du système semi-intensif (figure 2), une augmentation du prix des céréales pénalise le développement de la production laitière en volume $\mathrm{B}$. Cependant, une augmentation du prix du lait commercialisé en volume A influe positivement sur les volumes fournis en volume B. En effet, le Volume A est celui qui assure principalement la rentabilité économique de l'exploitation et sa pérennité, il ne peut $\mathrm{y}$ avoir de lait produit au-delà du Volume A que si son prix est suffisant et permet de couvrir les charges de structure de l'exploitation et de rémunérer la main-d'œuvre.

L'incitation à produire davantage de lait est faible lorsque le coefficient d'élasticité est élevé (supérieur à 3). En effet, dans ce cas, chaque pourcentage d'augmentation de la production laitière entraîne une réduction importante du prix du lait en volume B. A l'inverse, un coefficient plus faible aura pour conséquence de favoriser nettement la production laitière en volume $\mathrm{B}$, au détriment de la production céréalière ou bovine.

\section{Conclusion}

Ce modèle, basé sur la méthode de la programmation mathématique, a permis d'évaluer l'impact d'un scénario de sortie des quotas laitiers (parmi d'autres possibles) sur les stratégies productives des exploitations laitières. La prise en compte des interactions entre les productions animales et végétales, des principales lois de réponse biologique et de la saisonnalité de la production agricole permettent à ce modèle de représenter, de façon aussi réaliste que possible, le comportement des agriculteurs. Pour aussi développé qu'il soit, ce modèle pourrait encore faire l'objet d'améliorations, par exemple, pour intégrer d'autres objectifs au sein de la fonction d'optimisation (comme la réduction du temps de travail). Dans un contexte marqué par une forte volatilité des prix, la méthode utilisée, «Utility Efficient Programming», pourrait également être légèrement modifiée de façon à mieux intégrer les anticipations des agriculteurs face à l'orientation des variations de prix (positives ou négatives). De même, le nombre de cas-types pourrait être plus important afin de mieux représenter la diversité des systèmes de production. Un couplage de cette modélisation aux données du Réseau d'Information Comptable Agricole (RICA) pourrait aussi permettre d'étendre la portée de certains commentaires.

Les résultats de ces simulations montrent que l'instauration d'un système de type «double volume et double prix» pourrait inciter les producteurs de l'Ouest de la France à augmenter leur production laitière. De même, ils soulignent que les volumes de lait commercialisés en volume B seraient fortement

Figure 2. Influence du prix des céréales et du prix du lait en volume A sur la pro-
duction de lait en volume B simulée pour le cas-type d'exploitation "Semi-intensif».

Figure 2. Influence du prix des céréales et du prix du lait en volume A sur la pro-
duction de lait en volume B simulée pour le cas-type d'exploitation «Semi-intensif».

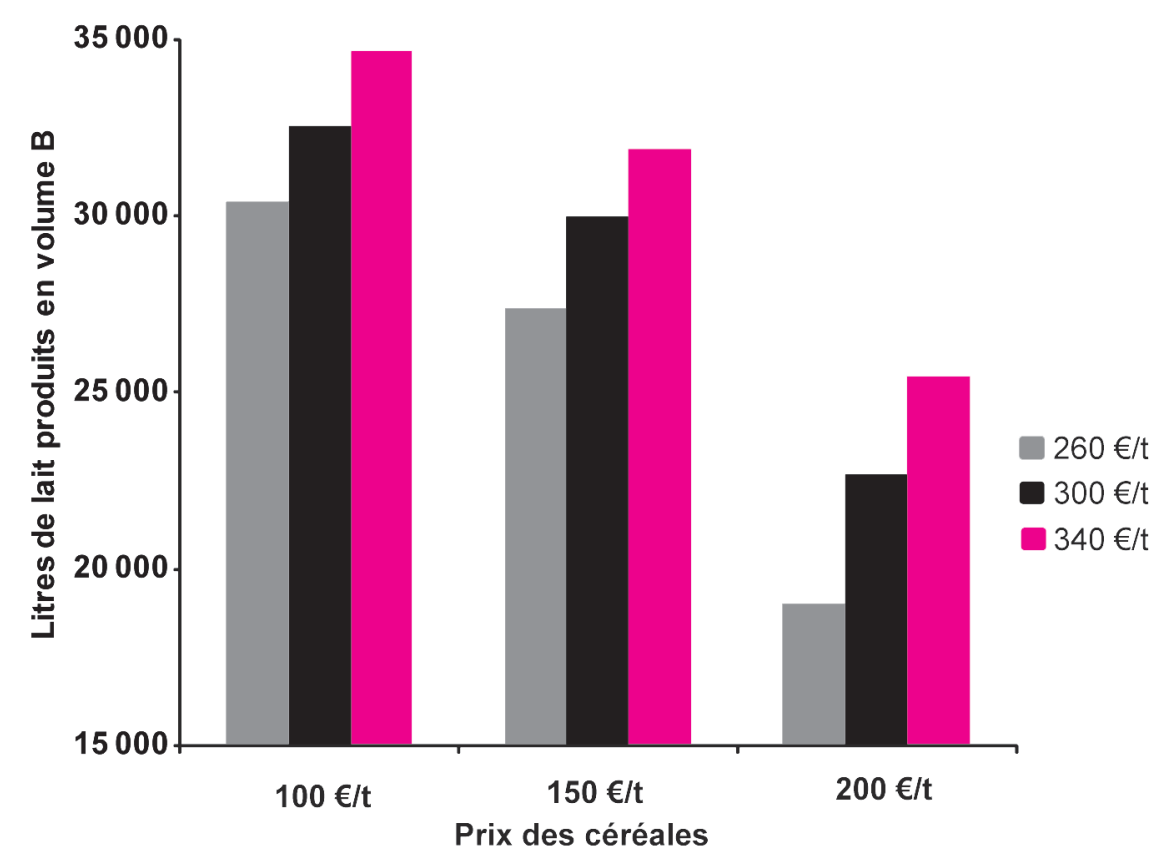

conditionnés aux rapports de prix entre les productions agricoles (par un effet demande à une augmentation des volumes de lait commercialisés. Avec la suppression du régime des quotas laitiers, le lien de la production laitière au foncier devrait être remis en cause, même si les normes environnementales joueront un rôle d'arbitrage sur les niveaux futurs d'intensification.

De plus, avec la suppression des quotas laitiers, la production laitière est susceptible de se concentrer davantage dans les bassins européens les plus compétitifs. Jusqu'alors la concurrence n'était que partielle dans la mesure où chacun disposait d'une protection de ses propres volumes au travers des quantités globales garanties. La localisation géographique future de l'offre est également susceptible d'évoluer en fonction des stratégies adoptées par les groupes industriels, au travers de leurs choix d'implantations et d'investissements. En l'absence de quotas, la recherche d'une diminution des coûts d'approvisionnement, pourrait en effet, inciter les transformateurs à favoriser certaines zones. L'obtention d'un coût de production compétitif constituera sûrement un atout pour permettre à l'agriculteur de négocier avec un industriel partenaire, une extension de ses volumes contracmême, les industriels auront intérêt à ce que les futurs volumes libérés (suite au départ en retraite des fournisseurs aînés) soient alloués au bénéfice des producteurs ayant des coûts de production de substitution) et à la réponse de la tualisés, ou pour changer de laiterie. De 
satisfaisants (ou en passe de le devenir moyennant une augmentation des volumes).

La suppression des quotas va modifier le cadre concurrentiel des entreprises européennes de la transformation laitière, et remettre encore davantage au centre des problématiques les questions relatives à l'innovation, à l'ouverture internationale et au mode de valorisation des produits laitiers. L'instauration d'une contractualisation avec les producteurs de lait est nécessaire pour permettre aux industriels de sécuriser leurs approvisionnements, en quantité et en qualité. Cependant, avec la mise en place de contrats, les entreprises de la transformation sont susceptibles de bénéficier d'un pouvoir renforcé en termes de fixation du prix du lait, d'exigences qualitatives, d'orientation des structures d'exploitations (taille, intensification) et de localisation territoriale de l'offre. Le passage d'une régulation publique (quota) à une régulation privée (contrat) suscite donc légitimement des inquiétudes de la part de nombreux producteurs. Au cours des prochaines années et d'ici à 2015, il convient sur- tout de bien définir, avec l'appui des pouvoirs publics et dans un cadre collectif, les termes précis de la future contractualisation.

\section{Remerciements}

Cet article a été réalisé dans le cadre du projet LAITOP du programme PSDR Grand-Ouest. Ces travaux de modélisation ont également bénéficié d'un appui financier du CNIEL.

\section{Références}

Ben Arfa N., Daniel K., Jacquet F., 2010. A generalized cross entropy approach to analyze the structural and spatial change in the French dairy farms. Journ. Rech. Sci. Soc. INRASFER-CIRAD. INRA, Rennes, France, p. 35

Berentsen P.B., Giesen G.J., Renkema J.A., 2000. Introduction of seasonal and spatial specification to grass production and grassland use in a dairy farm model. Grass Forage Sci., 55, 125-137.

Bewley J., Palmer R.W., Jackson-Smith D.B., 2001. Modeling milk production and labor efficiency in modernized wisconsin dairy herds. J. Dairy Sci., 84, 705-716.

Bouamra-Mechemache Z., Jongeneel R., Requillart V., 2009. Impact of a gradual increase in milk quotas on the EU dairy sector. Eur. Rev. Agric. Econom. 35, 461-491.

Brun-Lafleur L., Delaby L., Husson F., Faverdin P., 2010. Predicting the energy $x$ protein interaction on milk production and composition in dairy cows. J. Dairy Sci., 93, 4128-4143.

Chatellier V., Pflimlin A., Perrot C., 2008. La production laitière dans les régions de l'arc atlantique européen. INRA Prod. Anim., 21, 427-440.

Colman D., 2000. Inefficiencies in the UK milk quota system. Food Policy, 25, 1-16.
Commission Européenne., 2011. Proposition de règlement du Parlement Européen et du Conseil portant sur l'organisation commune des marchés des produits agricoles, Bruxelles, Belgique, $235 \mathrm{p}$.

Faverdin P., Delaby L., Delagarde R., 2007. L'ingestion d'aliments par les vaches laitières et sa prévision au cours de la lactation. In : Alimentation des ruminants. Agabriel J. (Ed). Dossier, INRA Prod. Anim., 20, 151162 .

Flaten O., Lien G., 2007. Stochastic utilityefficient programming of organic dairy farms. Eur. J. Oper. Res., 181, 1574-1583.

Godard C., Roger-Estrade J., Jayet P.A., Brisson N., Le Bas C., 2008. Use of available information at a European level to construct crop nitrogen response curves for the regions of the EU. Agric. Syst., 97, 68-82.

INRA., 2007. Alimentation des bovins, ovins et caprins : Besoins des animaux. Valeurs des aliments. Editions Quae, Versailles, France, 312 p.

INRA., University of Wageningen Consortium., 2002. Study on the impact of future options for the milk quota system and the common market organization for milk and milk products. Eur. Commission, 39p.
Institut de 1'Elevage., 2009. Les systèmes bovins laitiers en France - Repères techniques et économiques. Institut de l'Elevage, Paris, France, 32p.

Institut de l'Elevage., 2011. Stratégies d'approvisionnement des transformateurs laitiers après 2015. Dossier Economie de l'Elevage, 417, p68.

Kroll J.C., Trouvé A., Deruaz M., 2010. Quelle perspective de régulation après la sortie des quotas ? Faut-il encore une politique laitière européenne? Centre d'Economie et Sociologie appliquées à l'Agriculture et aux Espaces Ruraux (CESAER), Dijon, France.

Lelyon B., Chatellier V., Daniel K., 2011a. Impact of decoupling and price variation on dairy farmers' strategy. Overview of theoretical and real effects. Disaggregated impacts of CAP reforms: Proc. OECD Workshop. OECD, Paris, France, 111-131.

Lelyon B., Daniel K., Chatellier V., 2011b. Decoupling and prices: determinant of dairy farmers' choices? Rev. Agric. Environ. Stud., 92, 47-68.

Roux G., 2011. Recensement Agricole 2010 : premières tendances. Agreste Primeur, 266, 1-4.

\section{Résumé}

Les quotas laitiers, instaurés en 1984, seront supprimés à horizon de 2015. A partir d'un modèle de type bioéconomique, cet article discute des principales implications productives et économiques de cette évolution importante de la Politique Agricole Commune (PAC). L'hypothèse est faite que, concomitamment à l'abandon des quotas laitiers, un système de contractualisation de type «double volume avec des prix différenciés» sera mis en œuvre entre les producteurs et les industriels. Le modèle utilisé a pour objectif de maximiser le revenu de l'exploitation tout en tenant compte d'un ensemble de contraintes, qu'elles soient zootechniques, agronomiques, environnementales, structurelles et réglementaires. Ce modèle, qui permet d'intégrer le risque lié à la volatilité des prix, est appliqué à quatre types d'exploitations reflétant une partie de la diversité des systèmes de production localisés dans l'Ouest de la France. D'après les résultats issus de cette modélisation, il apparaît que de nombreuses exploitations laitières de l'Ouest disposent, moyennant une modification de leurs stratégies productives (intensification, spécialisation), d'un potentiel assez important d'augmentation de la production laitière, à structures constantes. La quantité de lait supplémentaire susceptible d'être produite dans cette région dépendra de l'évolution de la demande de produits laitiers, de la compétitivité des industries laitières et des normes environnementales. Pour une exploitation donnée, ces quantités supplémentaires sont également conditionnées au prix de vente du lait produit dans les deux volumes contractualisés et aux prix relatifs entre les produits agricoles (lait, viande bovine et céréales). 


\begin{abstract}
Production strategies after the end of milk quotas: an analysis based on bio-economic modeling

Milk quotas, introduced in France in 1984, will be ended by 2015. This paper, based on a "bio-economic" model, discusses the main productive and economic implications of this important change of the Common Agricultural Policy (CAP). We assumed that, with the abolishment of milk quotas, a contracting system of "double volume with different prices" will be implemented between producers and dairy processors. The model maximizes the farm income while taking into account a set of constraints: zootechnical, agronomical, environmental, structural and regulatory. This model, which integrates the risk of price volatility, is applied to four types of farms reflecting a part of the farms' diversity located in the west of France. According to the results of this modeling approach, it appears that many dairy farms located in this region have, thanks to changes in their production strategies (intensification, specialization), a large potential to increase milk production, with the same structure. The amount of extra milk which may be produced will depend on the evolution of demand for dairy products and the competitiveness of the dairy industries. For a given farm, these additional quantities depend on the price of milk produced in volume A and B, the relative prices between agricultural products (milk, beef and cereals) and a possible strengthening of environmental constraints.
\end{abstract}

LELYON B., CHATELLIER V., DANIEL K., 2012. Fin des quotas laitiers, contractualisation et stratégies productives : enseignements d'une modélisation bioéconomique. INRA Prod. Anim. 25, 67-76. 